\title{
An Improved Impedance/Admittance Analysis Method Considering Collector Subsystem Transformation in Converter-integrated Power Systems
}

\author{
Wang, Chenxuan; Wang, Zhen; Wu, Qiuwei; Xin, Huanhai
}

Published in:

IEEE Transactions on Power Systems

Link to article, DOI:

10.1109/TPWRS.2021.3107715

Publication date:

2021

Document Version

Peer reviewed version

Link back to DTU Orbit

Citation (APA):

Wang, C., Wang, Z., Wu, Q., \& Xin, H. (2021). An Improved Impedance/Admittance Analysis Method

Considering Collector Subsystem Transformation in Converter-integrated Power Systems. IEEE Transactions on Power Systems, 36(6), 5963-5966. https://doi.org/10.1109/TPWRS.2021.3107715

\section{General rights}

Copyright and moral rights for the publications made accessible in the public portal are retained by the authors and/or other copyright owners and it is a condition of accessing publications that users recognise and abide by the legal requirements associated with these rights.

- Users may download and print one copy of any publication from the public portal for the purpose of private study or research.

- You may not further distribute the material or use it for any profit-making activity or commercial gain

- You may freely distribute the URL identifying the publication in the public portal 


\title{
An Improved Impedance/Admittance Analysis Method Considering Collector Subsystem Transformation in Converter-integrated Power Systems
}

\author{
Chenxuan Wang, Zhen Wang, Member, IEEE, Qiuwei Wu, Senior Member, IEEE, Huanhai Xin, Member, IEEE
}

\begin{abstract}
To analyze oscillation phenomenon caused by the interaction between converters and an $\mathrm{AC}$ grid, the impedance/admittance analysis method is a popular solution, in which each electrical component is represented by an external impedance/admittance matrix and a lumped matrix is formed for stability analysis. However, for converter-integrated power systems considering typical collector subsystems (e.g., the radial and ring collector subsystem in wind farms and PV stations), the impedance network reduction to form the lumped impedance/admittance matrix (LIM/LAM) becomes complicated particularly for a ring collector subsystem, which affects the efficiency of the oscillation analysis. To address this problem, this paper proposes a method that can transform a typical topology of collector subsystems into a pure-parallel topology so that lumped admittance matrix is much easier to be derived. Algorithm complexity analysis confirms its computational advantages. A fifty-converter system is used to verify the effectiveness of the proposed method.
\end{abstract}

Index Terms-Converter-integrated power system, collector subsystem transformation, lumped impedance/admittance matrix (LIM/LAM), oscillation analysis

\section{INTRODUCTION}

$\mathrm{T}_{\mathrm{p} e \mathrm{es}}^{\mathrm{h}}$ he oscillation phenomenon is an important issue as the penetration of power electronics interfaced sources increases rapidly ${ }^{[1]}$. In recent years, the unstable oscillation events has been reported in many renewable energy integrated power systems, for example, the wind farms in Texas, USA and Xinjiang, China. Therefore, the oscillation instability risk evaluation and analysis becomes an urgent requirement for power system operation and planning.

To address this problem, a type of impedance/admittance (I/A) model based methods are widely adopted ${ }^{[2,3]}$, in which:

(1) Each electrical component (e.g., converters, transformers, and grid impedance) is represented by an external I/A matrix and an I/A network (INM/ANM) is formed according to the parallel/series circuit topology ${ }^{[4]}$;

(2) The INM/ANM is further reduced into a lumped I/A matrix (LIM/LAM) for further frequency-domain stability evaluation by some popular analysis methods, e.g., Bode stability criteria, Nyquist stability criteria, and pole-zero analysis ${ }^{[1]}$.

In most existing I/A analysis methods, the topology of collector subsystems of actual wind farms/PV stations is usually neglected. Instead, the wind farm/PV station is regarded as a single equivalent WTG/PV unit, because the algorithm complexity when forming the LIM/LAM in (2) will be greatly increased with these collector subsystems taken into account. In the early development of wind farms, the radial

This work is supported by the National Nature Science Foundation of China (No. 52077196 and No. 51922094) and the China Scholarship Council. (Corresponding author: Zhen Wang.)

C. Wang, Z. Wang and H. Xin are with the College of Electrical Engineering, Zhejiang University, Hangzhou 310027, China (e-mail: 11710035@zju.edu.cn; z.wang@zju.edu.cn; xinhh@zju.edu.cn).

Q. Wu is with the Department of Electrical Engineering, Technical University of Denmark, Lyngby 2800, Denmark (e-mail: qw@elektro.dtu.dk). collector subsystem is widely adopted for multiple WTG/PV units aggregation and the LIM/LAM calculation methods tailored for the radial collector topology are developed accordingly, e.g., the impedance separation method reported in [5]. However, as the ring topology is increasingly used to raise the collector's reliability against cable faults, the existing radial topology oriented methods can not be applicable any more.

To this regard, this paper proposes a collector subsystem topology transformation method, which can handle not only the radial topology but also the ring topology. A collector subsystem can be transformed into a pure-parallel topology, by which the INM/ANM can be easily lumped, and the algorithm complexity of LIM/LAM forming is quantitatively evaluated. In this paper, the admittance modeling will be used seeing that a nodal admittance matrix is much easily obtained.

\section{ADMITTANCE ANALYSIS OF CONVERTER-INTEGRATED POWER SYSTEMS}

\section{A. Converter-integrated power system with collector subsystems}

A typical power grid with an integrated collector subsystem is illustrated in Fig. 1 (a), where a number of full-power converters (e.g., type-4 wind farms, PV stations) are fed into a typical collector subsystem through some step-up transformers $\left(R_{T}+j \omega_{0} L_{T}\right)$ and finally integrated into an external equivalent grid (a voltage source $E_{g}$ plus an impedance $R_{g}+j \omega_{0} L_{g}$ ) through the point of common coupling (PCC).

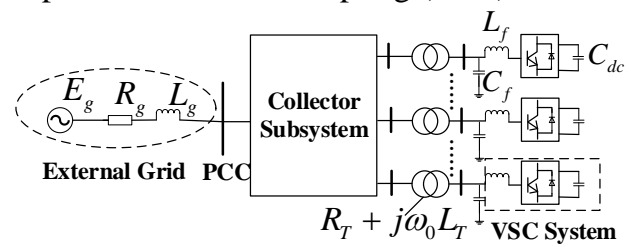

(a) Layout of a converter-integrated power system

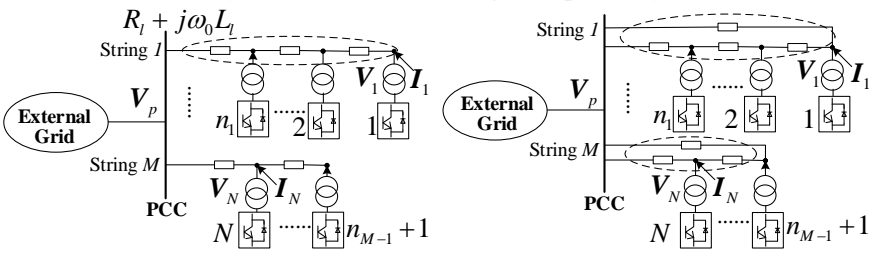

(b) Radial collector subsystem

(c) Ring collector subsystem

Fig. 1 Converter-integrated power system

Depending on the connection topology, typical collector subsystems can be categorized into two types: radial collector subsystem and ring collector subsystem. Fig. 1 (b) represents a radial collector subsystem (dotted-circle marked) connected with an external grid, where $N$ converters are grouped into $M$ parallel strings and connected in series using transmission cables. Fig. 1 (c) illustrates another ring collector subsystem, in which all converters are fed into PCC via a ring cable.

\section{B. Admittance network}

The admittance $\boldsymbol{Y}(s)$ of each component in Fig. 1 (b)-(c) is 
modeled under a unified $\mathrm{x}-\mathrm{y}$ synchronous rotating frame. Obviously, the terminal voltage deviation $\Delta \boldsymbol{V}=\left[\begin{array}{ll}\Delta V_{x} & \Delta V_{y}\end{array}\right]^{T}$ and the output current deviation $\Delta I=\left[\begin{array}{ll}\Delta I_{x} & \Delta I_{y}\end{array}\right]^{T}$ satisfies:

$$
\Delta \boldsymbol{I}=\left[\begin{array}{l}
\Delta \boldsymbol{I}_{x} \\
\Delta I_{y}
\end{array}\right]=\left[\begin{array}{ll}
Y_{x x}(s) & Y_{x y}(s) \\
Y_{y x}(s) & Y_{y y}(s)
\end{array}\right]\left[\begin{array}{l}
\Delta V_{x} \\
\Delta V_{y}
\end{array}\right]=\boldsymbol{Y}(s) \Delta \boldsymbol{V}
$$

where $\boldsymbol{Y}(s)$ is a s-domain admittance matrix with its $2 \times 2$ rational-function elements. For example, the $\boldsymbol{Y}(s)$ matrices for the transformers and the filter capacitors are:

$$
\boldsymbol{Y}_{T}=\left[\begin{array}{cc}
R_{T}+s L_{T} & -\omega_{0} L_{T} \\
\omega_{0} L_{T} & R_{T}+s L_{T}
\end{array}\right]^{-1} \quad \boldsymbol{Y}_{C}=\left[\begin{array}{cc}
s C_{f} & -\omega_{0} C_{f} \\
\omega_{0} C_{f} & s C_{f}
\end{array}\right]
$$

where $\omega_{0}$ denotes the fundamental angular frequency, and other parameters can be found in Fig. 1. According to the I/A analysis methods, an admittance network can finally be formed based on all individual admittance models ${ }^{[4]}$, as shown in Fig. 2 (a)-(b). In Fig. 2, the admittance $\boldsymbol{Y}_{V S C}$ of each converter will be firstly constructed based on a local d-q frame determined by the phase-locked loop (PLL), and then be transformed into an unified $x-y$ frame in the system ${ }^{[4]}$.

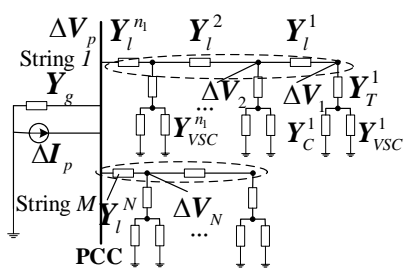

(a) Radial collector subsystem

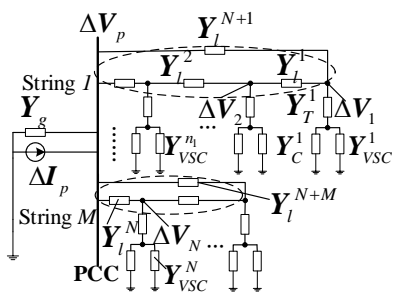

(b) Ring collector subsystem

\section{Pure-parallel collector subsystem transformation}

The admittance network reduction should be further conducted to form a lumped admittance matrix $\boldsymbol{Y}_{L A M}(s)$. However, $\boldsymbol{Y}_{L A M}(s)$ involves a series of parallel/series circuits simplification, which becomes very complicated for those real converter-integrated power systems with tens of collector subsystems included. To reduce the simplification complexities, the two typical topologies of collector subsystems in Fig. 2 (a)-(b) are considered to be transformed into a pure-parallel topology which is easier for LAM derivation ${ }^{[5]}$. The layout of the pure-parallel topology and the corresponding admittance network is illustrated in Fig. 3 (a)-(b) respectively, where the $i$ th converter subsystem is connected to PCC via an equivalent impedance $R_{l}^{i}+j \omega_{0} L_{l}^{i}$, which meets the following relationship:

$$
\left[\begin{array}{c}
I_{i x} \\
I_{i y}
\end{array}\right]=\left[\begin{array}{cc}
R_{l}^{i} & -\omega_{0} L_{l}^{i} \\
\omega_{0} L_{l}^{i} & R_{l}^{i}
\end{array}\right]^{-1}\left[\begin{array}{c}
V_{i x}-V_{p x} \\
V_{i y}-V_{p x}
\end{array}\right] \Leftrightarrow \boldsymbol{I}_{i}=\boldsymbol{Y}_{l}^{i} \cdot\left(\boldsymbol{V}_{i}-\boldsymbol{V}_{p}\right)
$$

where $\boldsymbol{V}_{p}=\left[\begin{array}{ll}V_{p x} & V_{p y}\end{array}\right]^{T}, \boldsymbol{V}_{i}=\left[\begin{array}{ll}V_{i x} & V_{i y}\end{array}\right]^{T}, \boldsymbol{I}_{i}=\left[\begin{array}{ll}I_{i x} & I_{i y}\end{array}\right]^{T}$ denote the steady-state PCC voltage, the $i$ th converter terminal voltage and its output current in vector form, respectively.

Accordingly, $\boldsymbol{Y}_{L A M}(s)$ can be easily derived as:

$$
\begin{gathered}
\boldsymbol{Y}_{L A M}(s)=\sum_{i=1}^{N} \boldsymbol{Y}_{L A M}^{i}(s) \\
\boldsymbol{Y}_{L A M}^{i}(s)=\left[\boldsymbol{Y}_{C}^{i}(s)+\boldsymbol{Y}_{V S C}^{i}(s)\right] \oplus \boldsymbol{Y}_{T}^{i}(s) \oplus \boldsymbol{Y}_{l}^{i}(s) \\
\boldsymbol{Y}_{1}(s) \oplus \boldsymbol{Y}_{2}(s)=\left[\boldsymbol{Y}_{1}^{-1}(s)+\boldsymbol{Y}_{2}^{-1}(s)\right]^{-1}
\end{gathered}
$$

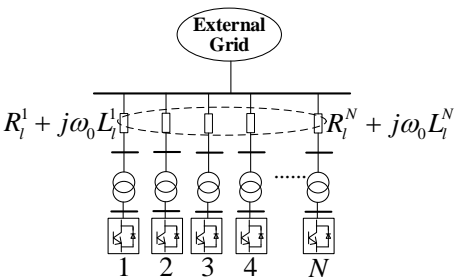

(a) Pure-parallel collector subsystem

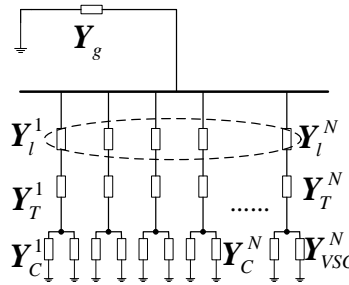

(b) Admittance network
Fig. 3 Pure-parallel collector subsystem and its admittance network

Usually, this converter-integrated power system is small-signal stable if the determinant polynomial of $\boldsymbol{Y}_{L A M}(s)$ doesn't have any right-half-plane roots. Considering $\boldsymbol{Y}_{L A M}(s)$ being a high-order polynomial, a frequency-scanning method can be applied to shape the $\boldsymbol{Y}_{L A M}(s)-\omega$ profile over $\omega \in\left[0, \omega_{\max }\right]$ and the system stability can be determined by evaluating the $\boldsymbol{Y}_{L A M}(s)$ matrix ${ }^{[4]}$.

\section{ADMITTANCE AGGREGATION AND ALGORITHM COMPLEXITY}

In a collector subsystem connecting $N$ converters with different topologies, the admittance aggregation process and the algorithm complexity at each frequency point is analyzed as follows:

(1) Radial collector: A series of parallel/series operations between neighboring admittances are conducted for every whole string in Fig. 2 (a). For example, the aggregated admittance $\boldsymbol{Y}_{s}$ for the first string can be derived as:

$$
\begin{aligned}
& \boldsymbol{Y}_{s}^{i}=\left[\left(\boldsymbol{Y}_{C}^{i}+\boldsymbol{Y}_{V S C}^{i}\right) \oplus \boldsymbol{Y}_{T}^{i}\right] \oplus \boldsymbol{Y}_{l}^{i}, i=1 \\
& \boldsymbol{Y}_{s}^{i}=\left[\left(\boldsymbol{Y}_{C}^{i}+\boldsymbol{Y}_{V S C}^{i}\right) \oplus \boldsymbol{Y}_{T}^{i}+\boldsymbol{Y}_{s}^{i-1}\right] \oplus \boldsymbol{Y}_{l}^{i}, i=2, \cdots, n_{1}
\end{aligned}
$$

Then the $\boldsymbol{Y}_{L A M}$ matrix is:

$$
\boldsymbol{Y}_{L A M}=\boldsymbol{Y}_{g}+\sum_{k=1}^{M} \boldsymbol{Y}_{s}^{n_{k}}
$$

where $n_{k}$ is the largest number of converters on String $k$ $(k=1,2, \ldots, M)$. Obviously, (7)-(9) totally have $2 N$ " + " and $2 N$ “ $\oplus$ ” matrix operations. Since the “ $\oplus$ ” operation includes 3 times inverse operation and 1-time addition operation according to (6). Therefore, $6 \mathrm{~N}$ times inverse operations and $4 N$ times addition operations are required to finish (7)-(9). By applying the Gauss-Jordan elimination method, the computation complexity of the inverse operation on any $n \times n$ matrix will be $O\left[\left(4 n^{3}+9 n^{2}-7 n\right) / 6\right]^{[6]}$. Obviously, the computation complexity of the addition operation between any $n \times n$ matrices will be $O\left(n^{2}\right)$. Therefore, the algorithm complexity of LAM computation at each $\omega \in\left[0, \omega_{\max }\right]$ is $O(6 N \times 9+4 N \times 4)$ as there is $2 \times 2$ matrix structure in $\boldsymbol{Y}(s)$.

(2) Ring collector: The nodal admittance matrix of Fig. 2 (b) is: $\left[\begin{array}{c}\Delta \boldsymbol{I}_{p} \\ \hdashline \boldsymbol{0} \\ \vdots \\ \boldsymbol{0}\end{array}\right]=\left[\begin{array}{c:ccc}\boldsymbol{Y}_{0,0} & \boldsymbol{Y}_{0,1} & \cdots & \boldsymbol{Y}_{0, N} \\ \hdashline \boldsymbol{Y}_{1,0} & \boldsymbol{Y}_{1,1} & \cdots & \boldsymbol{Y}_{1, N} \\ \vdots & \vdots & \ddots & \vdots \\ \boldsymbol{Y}_{N, 0} & \boldsymbol{Y}_{1, N} & \cdots & \boldsymbol{Y}_{N, N}\end{array}\right]\left[\begin{array}{c}\Delta \boldsymbol{V}_{p} \\ \hdashline \Delta \boldsymbol{V}_{1} \\ \vdots \\ \Delta \boldsymbol{V}_{N}\end{array}\right]=\left[\begin{array}{cc}\boldsymbol{Y}_{A} & \boldsymbol{Y}_{B} \\ \boldsymbol{Y}_{C} & \boldsymbol{Y}_{D}\end{array}\right]\left[\begin{array}{c}\Delta \boldsymbol{V}_{p} \\ \Delta \boldsymbol{V}_{i n}\end{array}\right]$ 
where $\boldsymbol{Y}_{i, j}(i, j=1, \cdots, N)$ are $2 \times 2$ matrix blocks, and

$$
\begin{aligned}
& \boldsymbol{Y}_{0,0}=\boldsymbol{Y}_{g}+\left(\boldsymbol{Y}_{l}^{N+1}+\cdots+\boldsymbol{Y}_{l}^{N+M}\right)+\left(\boldsymbol{Y}_{l}^{n_{1}}+\cdots+\boldsymbol{Y}_{l}^{n_{M}}\right) \\
& \boldsymbol{Y}_{0, n_{k-1}+1}=-\boldsymbol{Y}_{l}^{N+k}, \boldsymbol{Y}_{0, n_{k}}=-\boldsymbol{Y}_{l}^{n_{k}}\left(n_{0} \triangleq 0, k=1,2, \cdots, M\right) \\
& \boldsymbol{Y}_{n_{k-1}+1, n_{k-1}+1}=\left(\left(\boldsymbol{Y}_{C}^{n_{k-1}+1}+\boldsymbol{Y}_{V S C}^{n_{k-1}+1}\right) \oplus \boldsymbol{Y}_{T}^{n_{k-1}+1}\right)+\boldsymbol{Y}_{l}^{n_{k-1}+1}+\boldsymbol{Y}_{l}^{N+k} \\
& \boldsymbol{Y}_{i, i}=\left(\left(\boldsymbol{Y}_{C}^{i}+\boldsymbol{Y}_{V S C}^{i}\right) \oplus \boldsymbol{Y}_{T}^{i}\right)+\boldsymbol{Y}_{l}^{i}+\boldsymbol{Y}_{l}^{i-1}\left(i \neq \forall n_{k-1}+1\right) \\
& \boldsymbol{Y}_{i, i+1}=-\boldsymbol{Y}_{l}^{i}\left(i \neq \forall n_{k}\right) \\
& \boldsymbol{Y}_{A}=\boldsymbol{Y}_{0,0}, \boldsymbol{Y}_{B}=\left[\boldsymbol{Y}_{0,1} \cdots \boldsymbol{Y}_{0, N}\right], \boldsymbol{Y}_{C}=\left[\boldsymbol{Y}_{1,0} \cdots \boldsymbol{Y}_{N, 0}\right]^{T} \\
& \boldsymbol{Y}_{D}=\left[\begin{array}{ccc}
\boldsymbol{Y}_{1,1} & \cdots & \boldsymbol{Y}_{1, N} \\
\vdots & \ddots & \vdots \\
\boldsymbol{Y}_{1, N} & \cdots & \boldsymbol{Y}_{N, N}
\end{array}\right], \Delta \boldsymbol{V}_{i n}=\left[\Delta \boldsymbol{V}_{1} \cdots \Delta \boldsymbol{V}_{N}\right]^{T}
\end{aligned}
$$

Other matrix blocks are all zero matrices. By eliminating $\Delta \boldsymbol{V}_{\text {in }}$, $\boldsymbol{Y}_{L A M}$ can be obtained by Kron reduction.

$$
\Delta \boldsymbol{I}_{p}=\left(\boldsymbol{Y}_{A}-\boldsymbol{Y}_{B} \boldsymbol{Y}_{D}^{-1} \boldsymbol{Y}_{C}\right) \Delta \boldsymbol{V}_{p}=\boldsymbol{Y}_{L A M} \Delta \boldsymbol{V}_{p}
$$

Here an inverse operation on a $2 N \times 2 N$ matrix is required. Therefore, the algorithm complexity of LAM is $O\left[\left(32 N^{3}+36 N^{2}-14 N\right)\right]$.

(3) Pure-parallel collector: According to (4)-(6), $4 N$ inverse and addition operations on $2 \times 2$ matrices are required respectively. The algorithm complexity of LAM is $O(4 N \times 9+4 N \times 4)$, which is obviously smaller than that of the radial and ring the collector subsystems

\section{SIMULATION VERIFICATION}

A power system with a 50-converter ring collector

\begin{tabular}{|c|c|}
\hline Types & Setting \\
\hline $\begin{array}{l}\text { Electrical } \\
\text { parameters (p.u.) }\end{array}$ & $\begin{array}{l}R_{g}=0.10, L_{g}=0.40 ; R_{l}=0.007, L_{l}=0.007 ; \\
L_{T}=0.03, R_{T}=0.0\end{array}$ \\
\hline Converter (p.u.) & $\begin{array}{l}H_{d c}(s)=1.1+27.5 / \mathrm{s} ; \quad H_{i}(s)=1+50 / \mathrm{s}, \quad H_{p l l}(s)=60+ \\
1400 / \mathrm{s} ; C_{d c}=0.0544, C_{f}=0.075, L_{f}=0.15\end{array}$ \\
\hline Initial data(p.u.) & $V_{f d}=1.0, V_{f q}=0.0, I_{d}=1.0, I_{q}=0, V_{d c}=1.0, I_{d c}=1.0$ \\
\hline 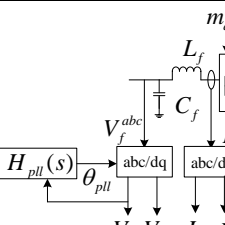 & $\begin{array}{l}{ }_{q}^{l_{q}} V_{d c} \\
\frac{1}{T} C_{d c} \oplus I_{d c}\end{array}$ \\
\hline
\end{tabular}
subsystem is used for simulation study, all identical converters are connected into two strings $(N=50, M=2)$. The basic AC voltage, DC voltage, and capacity of each converter is $575 \mathrm{~V}$, $1100 \mathrm{~V}$, and $2 \mathrm{MW}$ respectively. The system topology given in Fig. 1 (a) and (c) and the main data are listed in Table 1. The converter controller structure is illustrated in Fig. 4.

\section{Table 1 Main system data}

Fig. 4 Structure of converter controller

The disturbance is set as follows: Initially $E_{g}=1.0 \mathrm{pu}$; at $t=$ $0.2 \mathrm{~s} E_{g}$ drops to $0.95 \mathrm{pu}$ and recovers at $0.3 \mathrm{~s}$. The value of LAM is calculated at each frequency point over $0-100 \mathrm{~Hz}$ $\left(\omega_{\max }=200 \pi\right)$. A comparison study of the LAM frequency scanning with and without the proposed transformation on a
2.80GHz CPU/8GB RAM PC platform is conducted with different sampling intervals and the results is given in Fig. 5. Obviously, the proposed parallel transformation can improve computation efficiency compared no-transformation calculation (10)-(11). Further, the DC voltage of No. 25 converter and the dominant eigenvalues are presented in Fig. 6 (a) and (b), respectively, which attribute to subsynchronous oscillation (SSO) modes. As is observed in Fig. 6 (a), the time-domain simulation error is minor, and Fig. 6 (b) verifies that the transformed system is identical to the original system according to the SSO modes.
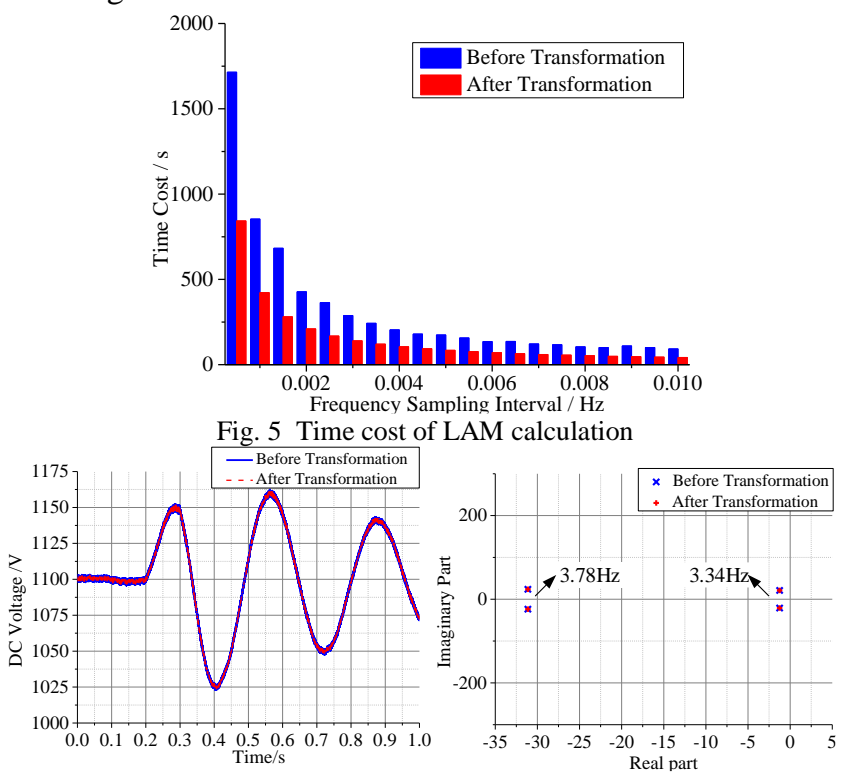

(a) The time-domain simulation result $\quad$ (b) The oscillation related eigenvalues Fig. 6 The oscillation characteristics before and after the transformation

\section{CONCLUSION}

A parallel transformation method for the collector subsystem in converter-integrated power system is proposed to reduce the algorithm complexity of LAM calculation. The time-domain simulation and the oscillation mode analysis reveal that the transformed system is identical to the original system.

\section{REFERENCES}

[1] L. Xu, H. Xin, L. Huang, H. Yuan, P. Ju, and D. Wu, "Symmetric admittance modeling for stability analysis of grid-connected converters," IEEE Trans. Energy Convers., vol. 35, no. 1, pp. 434-444, Mar. 2020

[2] J. Sun, "Impedance-based stability criterion for grid-connected inverters," IEEE Trans. Power Electron., vol. 26, no. 11, pp. 3075-3078, Nov. 2011.

[3] M. Cespedes and J. Sun, "Impedance modeling and analysis of grid-connected voltage-source converters," IEEE Trans. Power Electron., vol. 29, no. 3, pp. 1254-1261, Mar. 2014

[4] H. Liu, X. Xie, and W. Liu, "An oscillatory stability criterion based on the unified dq-frame impedance network model for power systems with high-penetration renewables," IEEE Trans. Power Syst., vol. 33, no. 3, pp. 3472-3485, May 2018.

[5] Y. Jin, D. Wu, P. Ju, C. Rehtanz, F. Wu, and X. Pan, "Modeling of wind speeds inside a wind farm with application to wind farm aggregate modeling considering LVRT characteristic," IEEE Trans. Energy Convers., vol. 35, no.1, pp. 508-519, Mar. 2020.

[6] R. W. Farebrother, Linear Least Squares Computations. New York, USA: Marcel Dekker, 1988 